\title{
Experimental suprachoroidal plombage with a urethane based hydrophilic polymer
}

\author{
W S FOULDS, DOROTHY AITKEN, AND W R LEE \\ From the Tennent Institute of Ophthalmology and Department of Pathology, University of Glasgow
}

SUMmARY Small portions of dehydrated hydrophilic polymer (HPU 90, Smith and Nephew) have been inserted into the suprachoroidal space in rabbits to investigate the possible use of suprachoroidal plombage in retinal detachment surgery. As it hydrates, the material causes a pronounced elevation of choroid and retina, and the implants have been well tolerated for periods of up to one year in experimental animals. The implant slowly breaks down and stimulates a simple macrophagic reaction: fibrosis in the overlying choroid is associated with ischaemic gliosis and chorioretinal fusion.

Localised indentation of the wall of the globe has an accepted role in the treatment of retinal detachment. The main function of such an indent is thought to be approximation of the choroid and retinal pigment epithelium (RPE) to the area of retina affected by a retinal hole. This allows the edges of the hole to be sealed on to the RPE and choroid by the induction of a localised inflammatory reaction by cyrotherapy, diathermy, or other means.

There are disadvantages in episcleral or intrascleral plombage. Distortion of the globe is a necessary result of the operation and may give rise to refractive problems or interfere with the action of an extraocular muscle, leading to diplopia. Extrusion of episcleral onlays is not an uncommon occurrence.

Redmond Smith in $1952^{1}$ suggested the use of suprachoroidal air as a way of achieving temporary choroidal elevation in retinal detachment surgery, but the technique was not widely adopted. One of us (WSF) investigated the use of suprachoroidal gelatin at that time, but preliminary experiments were not successful, mainly because it was found impossible to generate a localised indentation with this material. The advent of hydrophilic polymers offered the possibility of inserting a small portion of dehydrated polymer into the suprachoroidal space, relying on its subsequent hydration to cause a suitable elevation of the choroid.

This paper reports the results of experiments to investigate whether a hydrophilic polymer could be used for suprachoroidal plombage in experimental Correspondence to Professor W S Foulds, Tennent Institute of Ophthalmology, Western Infirmary, Glasgow G11 6NT. animals and to investigate the tissue response to see how well it would be tolerated.

\section{Materials and methods}

The material tested, HPU 90, was one of a series of hydrophilic urethane based polymers supplied for experimental purposes by Smith and Nephew. Samples were provided which in the hydrated state contained 85,90 , or $95 \%$ water. In the hydrated state all were translucent and of a soft, slightly elastic consistency. Dehydrated, they were firm and rigid.

\section{STERILISATION OF POLYMER}

Initial attempts to sterilise the material with ethylene oxide led to an unacceptable uveitis when the polymer was subsequently inserted into the eye, probably because the toxic ethylene oxide was retained in the polymer. Sterilisation with gamma radiation was found to cause degradation of the polymer, making it unsuitable for the purpose for which we intended to use it. Eventually the following method of sterilising the polymer was found satisfactory. Portions of hydrated ( $85 \%$ water content) polymer, $3 \mathrm{~mm} \times 1 \mathrm{~mm} \times 1 \mathrm{~mm}$ were continuously irrigated in distilled water for 24 hours to remove any leachables. The washed pieces of polymer were next placed in glass tubes sealed at either end with cottonwool plugs. The portions of polymer in the glass tubing were autoclaved at $134^{\circ} \mathrm{C}$ for seven minutes and subsequently dehydrated by being exposed to a current of dry sterile air drawn through the tubes via micropore filters type Millex-GS $0.22 \mu \mathrm{m}$ for a 
period of 12 hours. When dehydration was complete, one end of the tube was sealed and the other left sealed by the micropore filter.

\section{EXPERIMENTAL METHOD}

The experimental animals were Dutch pigmented rabbits of $1.9-2.9 \mathrm{~kg}$ body weight. The animals were anaesthetised with intramuscular chlorpromazine (BP) $(25 \mathrm{mg} / \mathrm{ml}, 0.5 \mathrm{ml} / \mathrm{kg}$ body weight $)$ and intramuscular ketamine hydrochloride $(50 \mathrm{mg} / \mathrm{ml}, 1 \mathrm{ml} /$ $\mathrm{kg}$ ). The conjunctiva in the upper temporal quadrant of one eye of each animal was incised and a $3 \mathrm{~mm}$ circumferential scleral incision made as posteriorly as was conveniently possible. The scleral incision was carried down to the suprachoroidal space, and an iris repositor was used to separate the choroid from the sclera just posterior to the incision. Through the scleral incision a portion of dehydrated polymer was inserted into the suprachoroidal space, care being taken not to damage the underlying choroid. The scleral incision was sutured by one or two $8 / 0$ virgin silk sutures. Topical gentamicin was instilled and the conjunctiva closed with one catgut suture.

\section{HISTOLOGICAL TECHNIQUE}

The animals were killed at various time intervals after insertion of the polymer and the eyes enucleated for histological examination, the unoperated eye being used as a control. Enucleated eyes were fixed in glutaraldehyde after a small incision had been made into the vitreous cavity through the pars plana to improve retinal fixation.

The eyes were examined macroscopically and photographed. Blocks were taken from the centre of the specimen, through the pupil and the optic nerve, across the centre of the implant, and were processed routinely for paraffin histology. Sections were stained with haematoxylin and eosin (H and $\mathrm{E})$, periodic acid Schiff (PAS), Bodian, Loyez, and Prussian blue for iron.

Tissue from selected eyes was postfixed with osmium tetroxide, dehydrated in alcohol, and embedded in Araldite for examination by transmission electron microscopy.

\section{Results}

The swelling characteristics of the polymer were tested in vitro by measuring the change in volume against time when a dehydrated portion of polymer was immersed in normal saline. Relative volume changes were assessed from measurements of the linear dimensions of the polymer sample by observing through a light microscope with an eyepiece graticule during hydration. The simple product of linear dimensions was taken rather than attempting

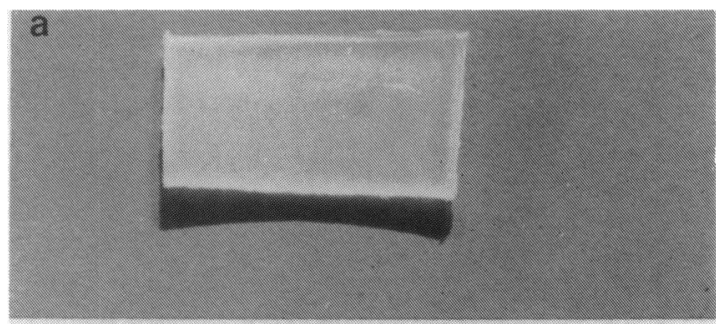

\section{Dehydrated Polymer}

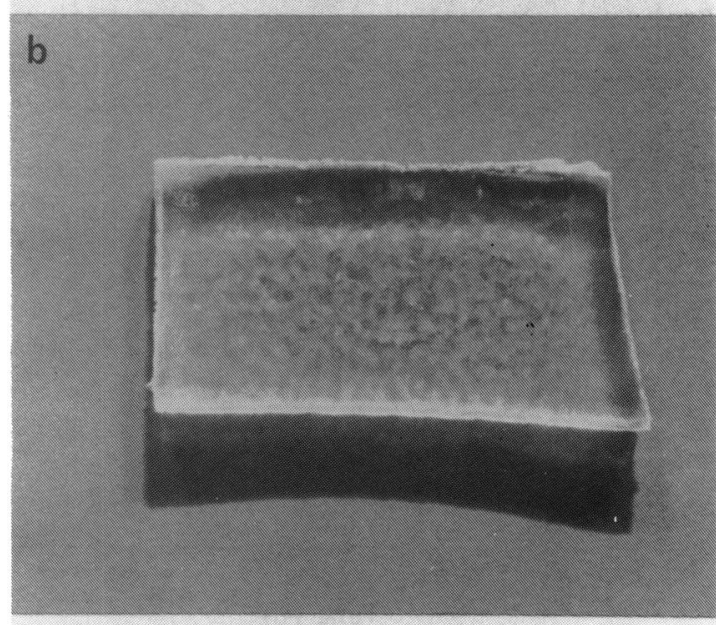

\section{Hydrated Polymer}

Fig. 1 Photographs of (a) dehydrated and (b) hydrated HPU 90 hydrophilic polymer to illustrate change in volume on hydration.

to account for irregularities of the specimen. The volume change with time is shown in Figs. 1 and 2, where it can be seen that hydration increased the volume to about 10 times that of the dehydrated state.

\section{SURGICAL RESULTS}

No great difficulty was experienced in inserting the dehydrated polymer into the suprachoroidal space. The material is rigid and easily handled when dehydrated, but if unsuccessful attempts are made to introduce it into the suprachoroidal space it rapidly starts to hydrate and quickly becomes pliable and slippery. It was found important to insert the material in one movement through the scleral incision and suture the incision quickly before significant hydration of the implant had occurred.

The material was inserted in nine rabbit eyes, and in none was there any evidence of immediate or delayed inflammation. Animals were killed for histological examination at time intervals after insertion of 


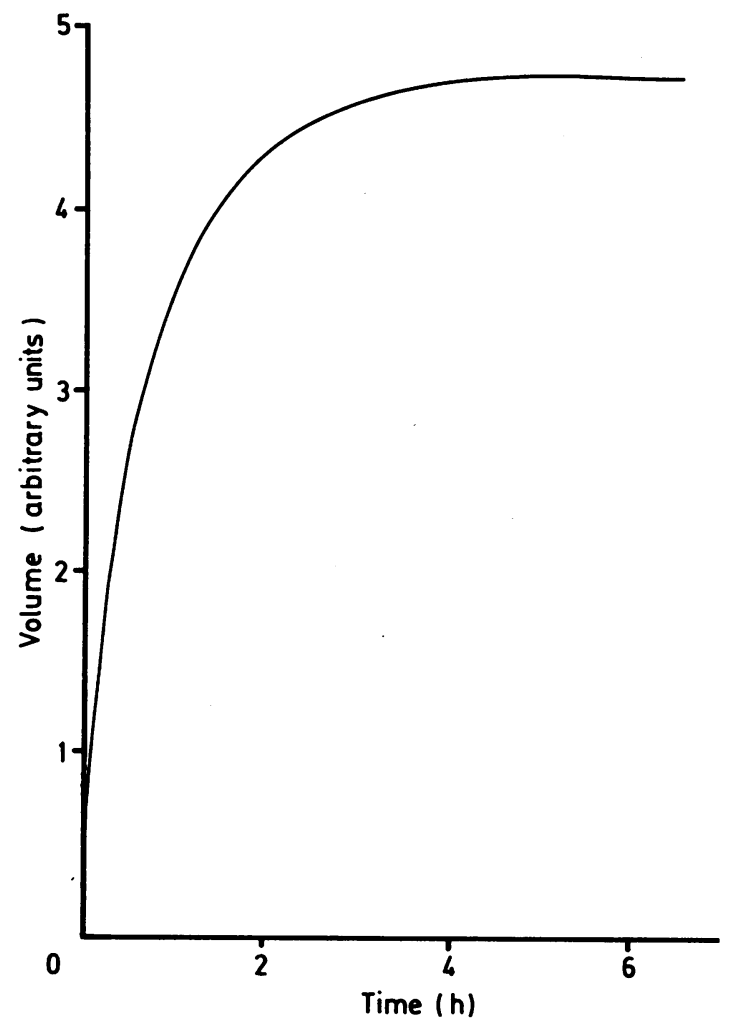

Fig. 2 Change in volume versus time when a dehydrated portion of HPU 90 polymer is rehydrated in vitro.

the polymer of one to two hours (four eyes), two weeks, two months, five months, 10 months, and 13 months.

Examination of the eyes immediately after surgery (Fig. 3) showed a localised elevation of choroid and retina which increased in size and depth over a period of two hours or so (Fig. 4). The rate of increase in size was established in one case by serial photography and measurements of height and breadth of the indent. The rate of increase in area of the indent appeared similar to that established for the isolated polymer in vitro. The only change noted in the appearance of the fundus over the months subsequent to the insertion of the polymer was a tendency for the development of a pigmentary disturbance in the region of the implant in two animals (Fig. 5) and a loss of pigment over the implant in another, so that a clear view of the sclera could be obtained through the implant. In one animal the height of the indent decreased after 10 months and by a year after insertion was almost flat (Figs. $6 a, b)$.

\section{HISTOLOGY}

On macroscopic examination all specimens of the

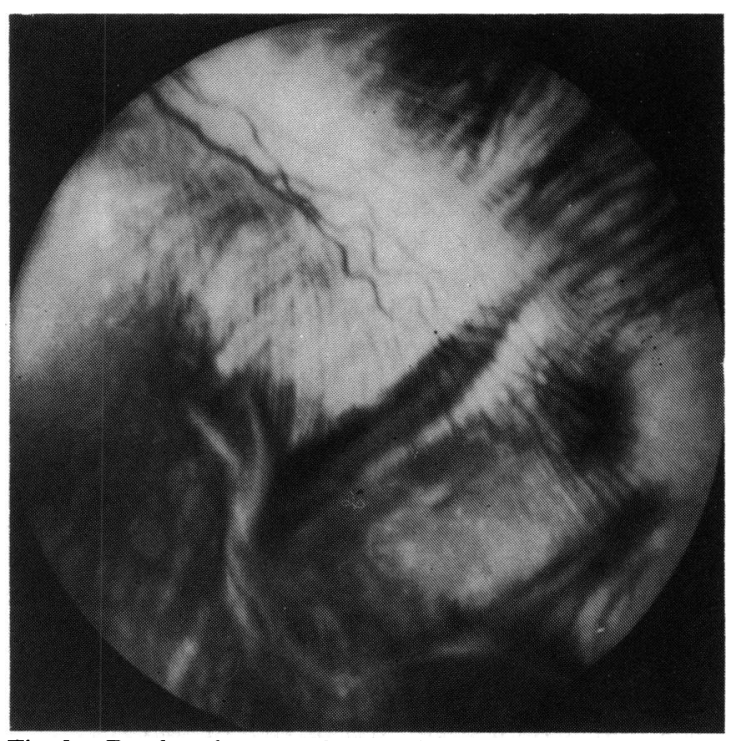

Fig. 3 Fundus photograph immediately after insertion of dehydrated HPU 90 into the suprachoroid to show localised elevation of choroid and retina.

eyes were free from significant inflammation in either anterior or posterior segment. The position of the implant was marked by a smooth localised elevation of the choroid and retina (Fig. 7). Transection of the implant revealed an empty space with choroid and retina elevated over it (Fig. 8). Histological examination of the tissues in the region of the implant showed a large empty space marking the site of the polymer

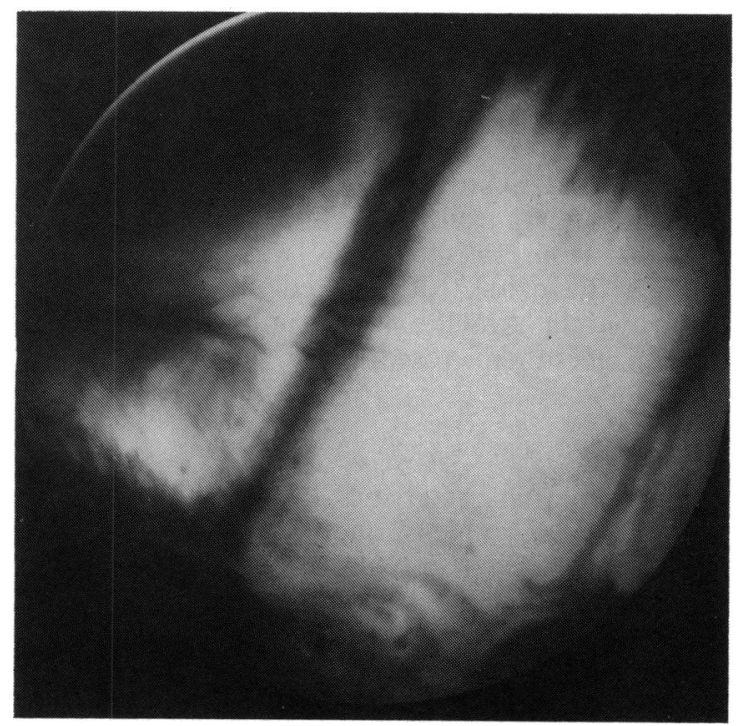

Fig. 4 Same eye as Fig. 3 two hours later to show increase in the size of the indent. 


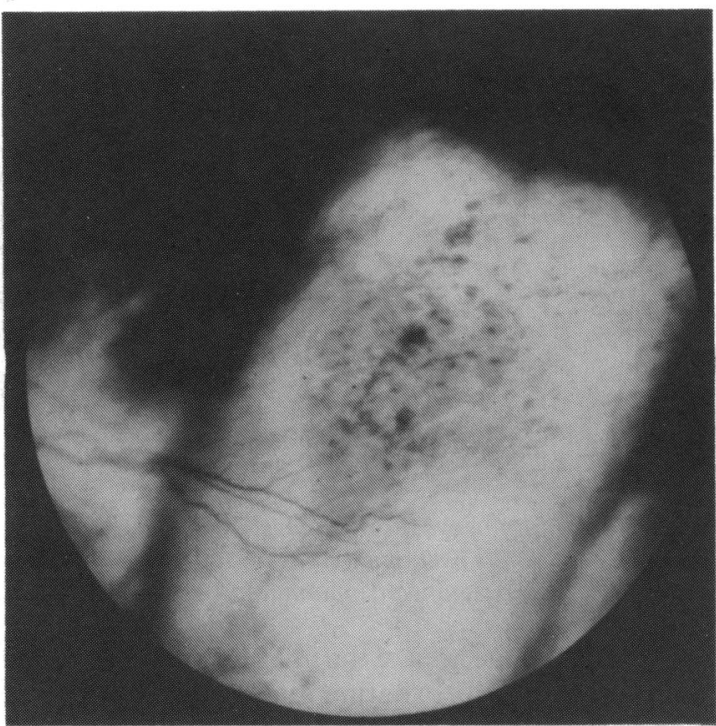

Fig. 5 Pigmentation over the implanted polymer in an eye in which the polymer had been present for eight weeks.

and overlying this a thinned choroid and intact but usually depigmented RPE and atrophic retina (Fig. 9).

In four globes in which the implant was in situ for 1-2 hours there was minor subretinal haemorrhage, with preservation of retinal architecture. The implant was located in the suprachoroidal space, and the overlying choroid was thinned. In two eyes there was

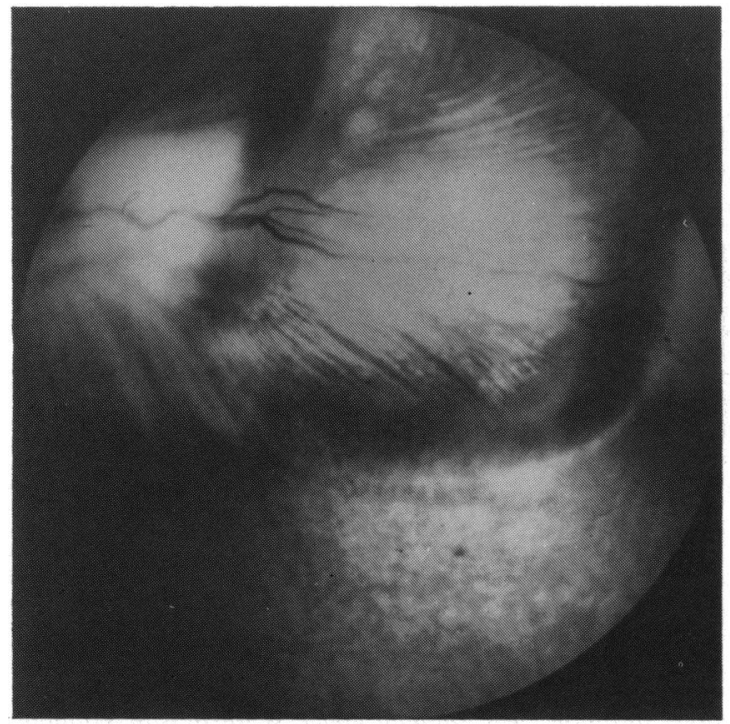

Fig. 6a

Fig. 6 Suprachoroidal implant at two months (a) and 10months (b). Height of the indent is decreasing. 


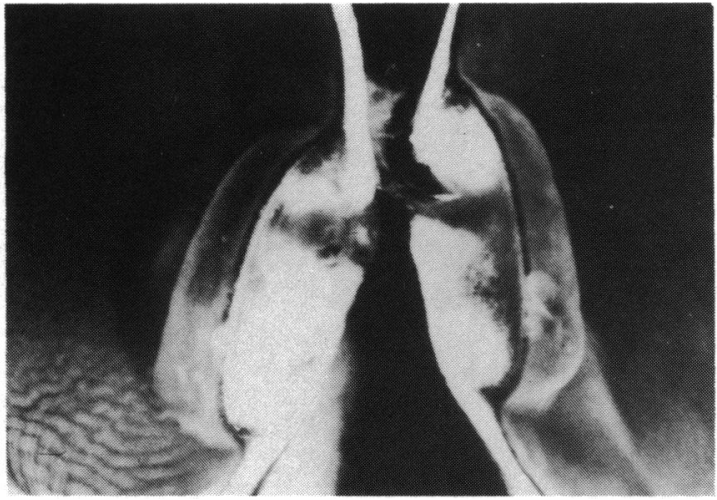

Fig. 8 Transection of the implant shown in Fig. 7 reveals the position of the implant as an empty space with choroid and retina elevated over it.

migration. The surrounding photoreceptor layer showed atrophy with reactionary proliferation of the RPE. In the 10-week specimen there was extensive retinal atrophy which extended up to $4 \mathrm{~mm}$ from the site of the implant. In each specimen the sclera beneath the implant showed a giant-cell granulomatous reaction to suture material, and in eyes in which the implant had been present for longer periods there was scleral thinning.

The globe removed at 10 months following implantation was different in that the inner retinal architecture was preserved and the RPE showed extensive reactionary proliferation. The globe removed 13 months after the insertion of the implant showed total resorption of the polymer; the site of implantation was recognised by thinning of the sclera, which contained clusters of foamy macrophages. Here there was fusion between a gliotic retina and a thin band of fibrotic choroid with an interposed layer of atrophic RPE.

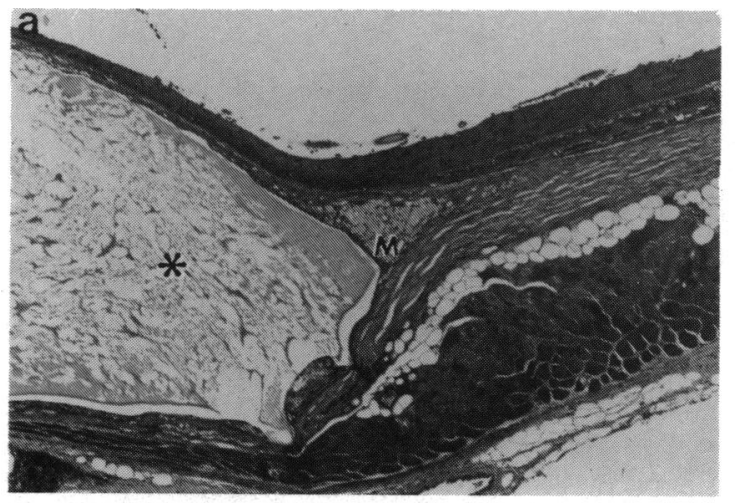

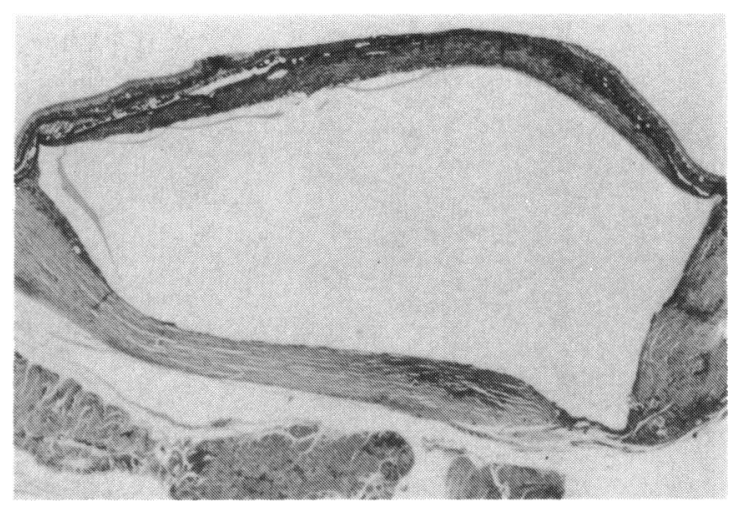

Fig. 9 Histology of eye shown in Figs. 7 and 8 . The implant is covered internally by thinned choroid, RPE, and atrophic retina. There is no significant inflammatory reaction. $\times 26$.

Apart from the area of the inlay all the other ocular structures in all experimental eyes showed no significant differences from the appearance of the control tissue.

\section{Discussion}

Suprachoroidal insertion of a small portion of dehydrated hydrophilic polymer is followed by hydration of the polymer in situ and the production of an elevation of the overlying choroid and retina. The depth of the indent is determined by the size of the initial implant but is approximately 10 times the volume of the dehydrated insert.

A suprachoroidal plombe of hydrophilic polymer is well tolerated in the rabbit eye and has caused no clinically unacceptable complications in a series of rabbits over periods of time up to one year. Nevertheless, the histological reactions are of interest. The most striking effect is the mononuclear macrophagic

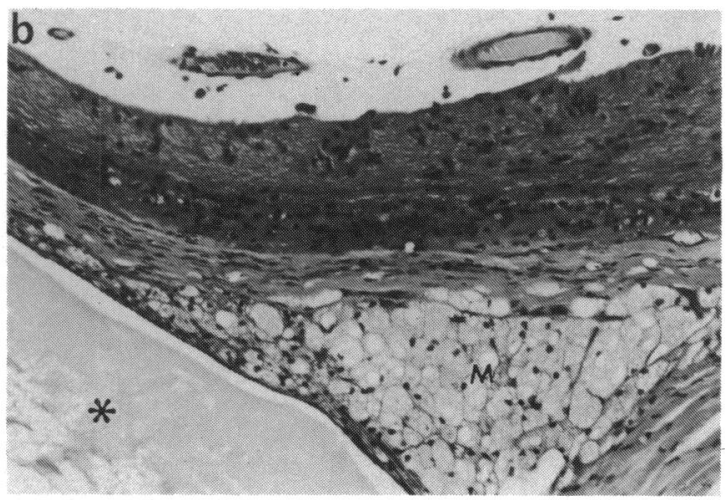

Fig. 10 Histology of a suprachoroidal implant of 4-5 months' duration. The implant space is filled with granular eosinophilic material $\left(^{*}\right)$ and surrounded by foamy macrophages $(\mathrm{M})$. The choroid is fibrotic and fused to the overlying RPE and retina. (a) $\times 26$. (b) $\times 110$. 
response to the polymer and the adjacent fibrosis in the choroid. The latter appears to be variable in extent. In some specimens choroidal fibrosis lead to total retinal atrophy, while in others there was preservation of the inner retina and reactionary proliferation of the RPE. The net effect in each specimen was that there was a broad zone of chorioretinal fusion similar to that resulting from therapeutically applied cryotherapy.

The use of this material as a suprachoroidal plombe in the management of human retinal detachment may be justified. A urethane based polymer in the form of a sponge has been successfully used as an episcleral indent, ${ }^{2}$ and another hydrophilic material has been used successfully as an episcleral or intrascleral inlay. ${ }^{34}$ In neither case has the material been used in the dehydrated state. The use of dehydrated polymer gives the advantage of a slowly enlarging buckle as hydration proceeds and consequent avoidance of raised intraocular pressure and embarrass- ment of the intraocular circulation which may occur when large changes in intraocular volume are suddenly produced by, for example, episcleral plombage with conventional techniques.

The authors thank Messrs Smith and Nephew for making the hydrophilic polymer available and Mrs Jennifer Murray for typing the manuscript.

\section{References}

1 Smith RJ. Suprachoroidal air injection for detached retina. Br J Ophthalmol 1952; 36: 385-8.

2 Kothe HW, Lommatzsch PK. Klinische Erfahrungen mit Polyurethan-Weichschaumplomben fur die Netzhautchirurgie. Klin Monatsbl Augenheilkd 1985; 187: 273-5.

3 Tolentino FI, Refojo MF, Schepans CI. A hydrophilic acrylate implant for scleral buckling, technique and clinical experience. Retina 1981; 1: 281-6.

4 Tolentino FI, Roldan M, Nassif J, Refojo MF. Hydrogel implant for scleral buckling. Long-term observations. Retina 1985; 5: $38-41$.

Accepted for publication 20 February 1987. 